Originalveröffentlichung in: Hartmut Günther und Otto Ludwig (Hg.), Schrift und Schriftlichkeit / Writing and Its Use, ... 1, Berlin und New York 1994, S. 289-297

19. Die ägyptische Hieroglyphenschrift und ihre Weiterentwicklungen

289

\title{
19. Die ägyptische Hieroglyphenschrift und ihre Weiterentwicklungen
}

1. Übersicht

2. Innere Form, dargestellt an der klassischen Bilderschrift

3. Äußere Form: Bilderschrift und daraus abgeleitete Kursivschriften

4. Entstehung

5. Einzelentwicklungen: Zeichenvorrat, Verhältnis Notation zu Kennzeichnung, Silbenschrift

6. Abhängigkeit anderer Schriften von der ägyptischen

7. Aussterben

8. Literatur

\section{1. Übersicht}

Die ägyptische Hieroglyphenschrift ist die Schrift des pharaonischen, vorchristlichen Ägyptens (zum Belegzeitraum siehe unter 4. und 7.). Als Zeichensystem ist sie vor allem durch zweierlei charakterisiert: Sie ist erstens eine teils semographische, teils phonographische Schrift. Sie zeigt zweitens ein komplexes Zusammenspiel zwischen der Notation sprachlicher Einheiten und der zusätzlichen Kennzeichnung dieser Notation (siehe unter 2.). Was die äußere Form angeht, bewegt sie sich zwischen den Polen einer - vorzugsweise monumentalen - Bilderschrift und Kursivschriften, denen in mehr oder minder hohem Grad die Bildhaftigkeit abgeht und in denen die Bedeutung der Einzelzeichen weit mehr als in der Bilderschrift hinter die der gesamthaften Zeichengruppen zurücktritt (siehe unter 3.). - Bei der Schrift-Entstehung sind Anstöße aus Sumer (Keilschrift) und/oder Elam nicht auszuschließen, die Schriftansätze entwickeln sich jedoch in Ägypten bereits sehr früh in anderer Weise als im zeitgleichen Vorderasien (siehe unter 4.). Die weitere Entwicklung des Schriftsystems ist gekennzeichnet durch Reduktion bzw. Ausbau des Zeichenvorrats, durch Neuordnung des Verhältnisses von Notation zu Kennzeichnung, eine Tendenz - namentlich in den Kursiven zur „Gruppenschreibung“ und durch Ansätze zur Schreibung der ursprünglich (und auch später im allgemeinen) nicht geschriebenen Vokale (siehe unter 5., zur Gruppenschreibung unter 3.). - Die Hieroglyphenschrift stand Pate bei der Entwicklung der westsemitischen Konsonantenschrift (proto-sinaitische bzw. proto-kanaanäische Schrift), sie lieferte die äußere Form der meroitischen Hieroglyphen- und Kursivschrift und einzelne Alphabetzeichen des griechisch-koptischen Alphabets der christlichen Ägypter (siehe unter
6.); die Frage eines möglichen Einflusses auf die Entwicklung von Silbenschriften vom Typ des Devanagari kann nicht abschließend entschieden werden, ist aber eher $\mathrm{zu}$ verneinen (siehe unter 5.3.).

\section{Innere Form, dargestellt an der klassischen Bilderschrift}

\subsection{Grundprinzipien}

Ihrer Funktion nach sind die hieroglyphischen Schriftzeichen(gruppen) in der Hauptsache entweder (1) Phonogramme oder (2) Semogramme. Beispiele: (1) Die Eule $f$ dient zur Notation des konsonantischen Phonems $m$, z. B. in der Schreibung der Präposition $m$ „in“; der Korb $\circlearrowleft$ zur Notation der Konsonantenfolge $n b$ (ohne Berücksichtigung eines etwa zwischen $n$ und $b$ stehenden Vokals), z. B. in der Schreibung des Quantors $n b$, jeder" (Genaueres unter 2.2.1.). (2) Der HausGrundriß $\square$ dient zur Notation des Wortes pr.w „Haus“ oder zur zusätzlichen Kennzeichnung der Notation von Bezeichnungen von Gebäuden und ihrer Teile, z. B. des Wortes - . (w)t „Kammer“, geschrieben etwa (Phonogramm ' + Phonogramm $t+$ Semogramm „Haus“ (Genaueres unter 2.2.2.). Neben Phonogrammen und Semogrammen gibt es (3) Schriftzeichen, denen innerhalb von Zeichengruppen nicht die Bedeutung zukommt, die sie als Einzelzeichen etwa hätten, oder die als Einzelzeichen keine Bedeutung haben, und (4) einzelne Schriftzeichen(gruppen), die der kalligraphischen Gestaltung der Hieroglyphenzeichen-Folgen oder als Interpunktion dienen. - Da Phonogramme - im allgemeinen nach dem Rebusprinzip - aus Semogrammen gewonnen sind, kann ein und dasselbe Hieroglyphenzeichen in beiderlei Funktion auftreten. Beispiel: $\square$ ist Semogramm „Haus“ und Phonogramm pr (aus pr.w „Haus").

Grundsätzlich können sprachliche Einheiten wie Morpheme, Wörter, Wortformen mit Semogramm oder mit Phonogramm(folgen) notiert werden. Beispiele: die Präposition $m$ „in“, geschrieben mit dem Phonogramm $\mathrm{m}$; das Wort $r n$ „Name“, geschrieben mit der Phonogrammfolge $\infty r+n$; das Wort $r^{\prime} w$ „Sonne, Tag“, geschrieben mit dem Semogramm ○, „Sonne“. Solche Schreibungen sind 
jedoch nur bei bestimmten Morphemen und Wörtern oder in bestimmten Wortverwendungen üblich. Im allgemeinen werden einem Teil oder allen der Notation dienenden Hieroglyphenzeichen andere Hieroglyphenzeichen der genannten Arten als Kennzeichnungen zugefügt: (1) Hieroglyphenzeichen(gruppen), die für eine Konsonantenfolge stehen, können durch Zufügung von Phonogrammen für eine initiale und/oder finale Teilkonsonantenfolge gekennzeichnet werden; Beispiele: $\triangle p r+r$

für die Phonogrammfolge $p r$, $\$$, śhm -Szep-

ter“ $+m$ für das Wort śhm ,mächtig sein“.

(2) Hieroglyphenzeichen(folgen), die für eine bedeutungstragende sprachliche Einheit, ein Morphem, ein Wort, eine Wortform stehen, können durch Zufügung von Semogrammen, die für alle oder einen Teil der semantischen Merkmale stehen, gekennzeichnet werden; Beispiele: $\Omega_{0} \circ$ Aी $r+{ }^{+}+$,Sonne“ + ,Verehrungswürdiger" für das Wort $r^{\prime}(w)$,(Sonnengott) Re“, $\odot \hat{s}$,Sonne“ + ,Verehrungswürdiger“ für das Wort $r^{\prime}(w)$,(Sonnengott) Re“. - Hieroglyphenzeichen(gruppen), die Phoneme notieren, werden üblicherweise schlicht als „Phonogramme“ bezeichnet, Hieroglyphenzeichen(gruppen), die bedeutungstragende Einheiten notieren, als „Logogramme“ oder „Ideogramme“. Hieroglyphenzeichen(gruppen), die phonographisch kennzeichnen, werden üblicherweise als phonetische (besser wäre: phonographische) Komplemente oder kurz als Komplemente bezeichnet, Kennzeichnungen nach (2) als Determinative.

\subsection{Funktionen der Hieroglyphenzeichen im einzelnen}

2.2.1. Phonogramme gibt es für alle konsonantischen Phoneme der ägyptischen Sprache (Einkonsonantenzeichen) und für eine gröBere Anzahl von Folgen von zwei konsonantischen Phonemen (Zweikonsonantenzeichen). Beispiele: die Eule $\mathbb{A}$ für $m$; der Korb

für $n b$. Inwieweit es Phonogramme für längere Folgen konsonantischer Phoneme gibt, ist nicht leicht $\mathrm{zu}$ entscheiden (es gibt $\mathrm{zu}$ wenige Anwendungsfälle; $\mathrm{zu}$ derartigen Problemfällen siehe unter 2.2.3.). - Ob man die Phonogramme als Konsonantenzeichen versteht oder - mit Gelb 1963 - als Silbenzeichen, ist eine Frage der Theoriebildung. Folgt man Gelb, so ist zu beachten, daß es sich bei den Schreibsilben nur teilweise um Sprachsilben handelt, generell also um eine Art Buchstabiersilben.
2.2.2. Semogramme sind in der Hauptsache Darstellungen eines semantisch mehr oder minder eng mit dem sprachlich Bezeichneten zusammenhängenden Objekts. Die Skala reicht von - nach den Darstellungsprinzipien der ägyptischen Kunst - einigermaßen vollständigen Darstellungen des sprachlich Bezeichneten (Piktogramme, Champollions figurative Zeichen) über die Darstellung von dem sprachlich Bezeichneten nahestehenden Objekten bis hin $\mathrm{zu}$ rein konventionellen Symbolen (Champollions tropische Zeichen). Beispiele: Es stehen das Einzelobjekt Sonne, - (Sonnenscheibe mit distingierendem Punkt), für $r^{\prime} w$,Sonne, Sonnengott Re“, der (zur Zeit der Erfindung dieses Schriftzeichens typische) Hausgrundriß (Einraumhaus), $\square$, für pr.w „Haus“, das (in ältester Zeit übliche) Schreibgerät, $\alpha^{\prime \prime}$, für $s \underline{h}$ ’. $w$,Schreiber“, die Beine, $\Omega$

für $i$,kommen“; die hockende (männliche) Person, für die grammatische Kategorie 1. Person, die (grammatische) Person par excellence; zwei Striche, " , für śnw.wi ,zwei“ oder die grammatische Kategorie Dual, drei Striche, "I" , für hmt.w „drei“ oder die Pluralität (grammatische Pluralität, Kollektivbegriffe, nicht Abzählbares). - Bei der Zuordnung zwischen Sprache und Schrift kommt dem Bedeutungsumfang der sprachlichen Elemente und dem paradigmatischen (meist auch etymologischen) Zusammenhang eine bedeutende Rolle zu. Z. B. steht das Einzelobjekt Sonne, $\odot$, nicht nur für das Wort $r^{\prime} w$ im Sinne von „Sonne, Sonnengott Re“, sondern auch für eben dieses Wort im Sinne von „Tag“, oder es steht das Paarhuferbein, \&, nicht nur in der Schreibung des Wortes whm(.t) „Paarhufer", sondern - weitaus häufiger - in der Schreibung des diesem als Etymon zugrundeliegenden Verbums whm ,wiederholen“.

2.2.3. Es gibt Hieroglyphenzeichen, die im Einzelfall oder generell in einer Grauzone zwischen Semogramm und Phonogramm stehen. Beispiele: Der Neumond $\Theta * p s ́ c ̧ n$ o. ä., mit dem man außer einer Ableitung von diesem Wort kaum mehr etwas anderes als das Wort pśč́(.t) ,(Götter-)Neunheit" notiert; die Notation des Adjektivs (der sog. Nisba) n'tt ,zur Stadt gehörig" mit zwei Stadtzeichen, \& , d. h. als der gleich oder ähnlich klingende Dual $n$ 'ti ,,beide Städte“. In solchen Fällen ist das Prinzip am Werk, dem Phonogramme in der Regel ihre Entstehung verdanken, das Rebusprinzip: die Verwendung des mit einem Semogramm gekoppelten Lautwerts für die Notation der gleichen oder ähnlichen Konsonan- 
tenfolge in beliebigen Zusammenhängen. Während echte Phonogramme viele solche Anwendungen gefunden haben, blieben solche wie die genannten auf einzelne Anwendungsfälle beschränkt, stellen also eine Art Quasi-Semogramme dar. - Solche Quasi-Semogramme sind ferner Abkürzungen wie $f$ ś für śnb.w ,er sei gesund“ im formelhaften Ausdruck 'nh.w wč ’. $w$ śnb.w ,er lebe, sei heil und gesund" und traditionelle Schreibungen, die aus dem zeitgenössischen orthographischen System nicht mehr (voll) verstanden werden können, wie die Schreibung des Wortes für „König (von Oberägypten)“, ұょ , was zeitgemäß kaum anders denn als św(.)t gelesen werden kann, in Wirklichkeit aber als orthographische Lösung aus der Frühzeit der Schriftentwicklung — für $n s w$ steht.

2.2.4. Komplemente bzw. Komplementfolgen, die bis zur Mitte der Konsonantenfolge des $\mathrm{zu}$ komplementierenden Phonogramms bzw. Semogramms stehen, werden im Standardfall vor das betreffende Phonogramm bzw. Semogramm gesetzt. Komplemente bzw. Komplementfolgen, die ab der Mitte der Konsonantenfolge des zu komplementierenden Phonogramms bzw. Semogramms stehen, werden hinter das betreffende Phonogramm bzw. Semogramm gesetzt. Beispiele mit zweikonsonantiger Phonemfolge: 今 $t+t m$, $p+;, 4 \Leftrightarrow i+i n+n$. Die Wahl des Komple-

mentierungstyps ist in Grenzen frei, sie hängt von kalligraphischen Erwägungen, von der unter 3. behandelten Schriftart oder von zeitspezifischen Vorlieben ab (Weiteres hierzu unter 5.2.).

2.2.5. Determinative stehen hinter der Notation der zu kennzeichnenden Einheit. Beispiel: 도요 $[;, h(. t)$, ,Horizont“ $+t$ für das Wort ; h.t "Lichtland, Horizont“] + "Gebäude“ + $t w$ + "Verehrungswürdiger" + "Plural“ für die Wortform sh.tw „Horizontische (Götter)“. Es gibt spezielle Determinative, die die Bedeutung bestimmter Morpheme, Wörter oder Wortformen (und der davon abgeleiteten) angeben, und allgemeine (generelle, generische) Determinative, die ein semantisches Merkmal angeben, das mehr als einem Morphem, einem Wort oder einer Wortform (und der davon abgeleiteten) zukommt. Zeichen ersterer Art sind in der Regel auch als semographische Notationen (Logogramme/Ideogramme) in Gebrauch. Beispiele: (1) für spezielles Determinativ: $\Delta$,Pyramide“ in $7 \mathrm{~S} \Delta$ $m r+m+r$ (nach Inversion aus kalligraphischem Grund statt* * $0 m+m r+r)+$
„Pyramide“ für das Wort $m r$ „Pyramide“ (daneben die logographische Schreibweise wie z. B. $\triangle$ „Pyramide“ für das Wort $m r$ „Pyramide"); (2) für allgemeines Determinativ: „Gebäude“ in $\overrightarrow{\text { อก }}$ " $t+$,Gebäude“ für '.(w)t „Kammer“, 今 ; $h(. t)$,Lichtland, Horizont" $+t+$,Gebäude“ für ; h.t „Lichtland, Horizont" und viele andere Gebäude(teil)bezeichnungen. - Die allgemeinen Determinative bilden eine Art semantisches Klassifikationssystem. Es gibt „(männliche) Personen“ (hockender Mann 迎), „Frauen“ (hockende Frau \& ), ,Gebäude“ (Haus $\curvearrowleft$ ), „Fremdländer“ (Bergland $\infty$ ), „schlechte Dinge“ (Sperling s- ), „Abstraktes“ (Buchrolle $\approx$ ) und vieles mehr.

2.2.6. Die Graphie ist nicht genormt, aber auch wiederum keineswegs beliebig. Es herrscht eine Vorliebe für phonographische Notation plus semographische Kennzeichnung (Determinierung). Die semographische Notation (Logogramm, Ideogramm) ist nur eingeschränkt möglich, es gibt nur für eine beschränkte Anzahl von Wörtern (schätzungsweise um die tausend, in ständigem Gebrauch davon mehrere hundert) entsprechende Hieroglyphenzeichen, und auch in diesen Fällen wird die phonographische Notation vielfach alternativ angewandt. Semographische Kennzeichnung ist üblich, sie unterbleibt bei einigen kurzen, häufig gebrauchten Wörtern, so z. B. bei der Präposition $m$ „,in“ (aber auch merkwürdigerweise bei dem Wort rn „Name“), und nach Morphemen wie z. B. den Personalsuffixen der 2. und 3. Person Singular. Die Schreibkonventionen folgen bei unterschiedlichen Teilwortschätzen unterschiedlichen (unterschiedlich alten) Regeln. So werden z. B. Beamtentitel gerne ,archaisch"-knapp geschrieben. - Die Wahl unter alternativen Schreibweisen ist von diversen Faktoren abhängig, so vom Ko-Text, vom Beschreibmaterial, von kalligraphischen Rücksichten. Beispiel: $w^{\prime}, w$,einer“ kann kotext-abhängig evtl. mit dem ,hockenden Mann“, 通, als gewöhnlicher Sterblicher oder mit dem ,hockenden Mann mit Bart“, 요 , als „Verehrungswürdiger“ (Gott, König oder dgl.) gekennzeichnet werden.

\section{3. Äußere Form: Bilderschrift und daraus abgeleitete Kursivschriften}

3.1. Die Hieroglyphenschrift, ursprünglich eine „Bilderschrift“, hat auf Dauer ihre Bildhaftigkeit bewahrt $(\rightarrow$ Abb. 34.1 auf Tafel 
IV). Daneben entwickelte sich früh eine Kursivschrift, das sog. Hieratische. Auch wenn dessen Zeichenformen oft nicht mehr die ursprünglichen Bilder erkennen lassen, blieben den Ägyptern die Entsprechungen auf Dauer weitgehend geläufig. Eine Etappe in der Auseinandersetzung der Bilderschrift und der Kursivschrift wird im 7. Jahrhundert v. Chr. mit der sog. Demotischen Schrift und der ephemeren sog. Abnormal-Hieratischen Schrift erreicht, Schriften, bei denen es nicht mehr ohne weiteres und oft gar nicht mehr möglich ist, kursive Schreibungen in Bilderschriftzeichen zurückzuübersetzen. - Eine Rückentwicklung des Hieratischen in Richtung auf die Bilderschrift stellen die sog. Kursiv-Hieroglyphen dar, eine Kursive, deren Zeichenformen den hieroglyphischen wieder stärker angenähert sind (diese Schriftart wird auch, da in Totenbuchhandschriften angewandt, als Totenbuch-Hieroglyphen bezeichnet; $\rightarrow$ Abb. 34.2).

3.2. Die Grund-Schreibrichtung der Bilderschrift ist diejenige von rechts nach links (angewandt hier in Abb. 19.1). Die entgegengesetzte Schreibrichtung, von links nach rechts, ist sehr gebräuchlich, bedarf aber in aller Regel einer besonderen Motivierung (hier, wie in modernen Druckwerken üblich, im laufenden Text angewandt). Die Kursivschriften (ausgenommen die Kursiv-Hieroglyphen) besitzen nur die Standard-Schreibrichtung der Bilderschrift, diejenige also von rechts nach links. - Bilderschrift-Texte werden $\mathrm{zu}$ allen Zeiten entweder in (vertikalen) Kolumnen oder in (horizontalen) Zeilen geschrieben, wobei in älterer Zeit die Kolumnenschreibweise vorherrscht, in jüngerer Zeit - archaisierende Tendenzen außer acht gelassen - die Zeilenschreibweise. Hieratisch wird früh in Kolumnen, später und zunehmend in Zeilen geschrieben, Demotisch und Abnormal-Hieratisch standardmäßig nur noch in Zeilen.

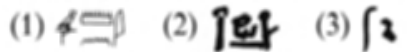

Abb. 19.1: Schreibung des Götternamens Amun in (2) hieratischer Schrift und (1) Re-Transkription in Bilderschrift (von rechts nach links bzw. von oben nach unten: i + mn + n +, ,Gott“; in echter Bilderschrift würde man nicht das angegebene Determinativ gebrauchen) sowie (3) in demotischer Verkürzung.

3.3. In der Bilderschrift können die Schriftzeichen infolge ihrer Bildhaftigkeit über ihre
Zeichenfunktion hinaus Bildfunktion haben. Das äußert sich vor allem in der Ausgestaltung der Bildzeichen selbst (Variation und Neuschaffung von Zeichenformen) und in der Ko- und Kontext-Sensibilität (Wahl und Kombination der Schriftrichtungen und der Blickrichtung der einzelnen Hieroglyphen; siehe Fischer (1977 a; 1986).

3.4. In den Kursiven verlieren die Zeichenformen an Selbständigkeit. Zeichenfolgen werden $\mathrm{zu}$ Ligaturen verbunden $(\rightarrow \mathrm{Abb}$. 34.3). Der fallweise eintretenden Verunklärung der Einzelzeichen wird entgegengewirkt durch eine reichere und stärker standardisierte Orthographie. Wichtiger als das Einzelzeichen wird die Zeichengruppe (,Schematogramm": Morphem-, Wort-, WortformSchreibung), zumal in den spätesten Kursiven des Demotischen und des ephemeren Abnormal-Hieratischen. Dabei verlieren auch die Determinative an Individual-Wert. Auf der anderen Seite hilft man sich im Demotischen dort, wo Wortschreibungen nicht tradiert sind, mit der Notation des zeitgenössischen Lautstands, ohne sich mit den älteren Komplementierungsusancen $\mathrm{zu}$ belasten, unter vorzugsweiser Verwendung von Einkonsonantenzeichen. Es vermehren sich auch rebusartige Ersatzschreibungen vom Typ n't $i^{3}$ (siehe unter 2.2.3.).

3.5. In Einzelfällen werden Kursivzeichen (in stilisierter Form) in die Bilderschrift übernommen. Beispiel: e $w$ (die kursive Form des Bilderschriftzeichens $\mathbb{w}$ ).

\section{Entstehung}

Die frühesten Schriftzeugnisse, die sich relativ-chronologisch sicher datieren lassen und deren Charakter als Schriftzeichen sich mit Bestimmtheit behaupten läßt, stammen aus der abydenischen Königsnekropole. Sie sind mit Königen der ,prädynastischen“ 0. Dynastie verbunden, und zwar bis zur fünften Generation vor die sog. Reichseinigung zurück, mit der die traditionelle Dynastiezählung einsetzt (Kaiser 1990). Absolut-chronologisch ist dieser Zeitpunkt nur approximativ festzulegen. Da die „Reichseinigung“ grob geschätzt um 3000 v. Chr. anzusetzen ist, reichen die Schriftzeugen sicher in das ausgehende 4. Jahrtausend zurück. Ob damit bereits die ersten Anfänge der Schrift in Ägyp- 
ten gefaßt sind, läßt sich nicht mit Bestimmtheit sagen. Grundsätzlich ist damit zu rechnen, daß damals — wie später — auf vergängliche, nicht erhalten gebliebene Materialien geschrieben wurde, nicht gerade auf Papyrus (der seit der 1. Dynastie - unbeschriftet - belegt ist), sondern etwa auf Baumblätter, die in späteren Ritualszenen als Schriftträger eine Rolle spielen. - Die frühesten Schriftzeugen sind schrift-systematisch meist schwer zu beurteilen, weil ihre Lesung unsicher oder unmöglich ist. Erst im Laufe der 1. Dynastie werden die Zusammenhänge mit der uns hier beschäftigenden Hieroglyphenschrift deutlicher: Es nehmen die Schriftzeichen zu, die man mit späteren Hieroglyphenzeichen identifizieren, d. h. lesen, kann, und im Gegenzug die Schriftzeichen ab, die im späteren Schriftzeicheninventar nicht mehr vorkommen, für uns daher im allgemeinen bislang unlesbar geblieben sind. Es lassen sich ferner, je später desto besser, die unter 2 . behandelten Schriftprinzipien erkennen, die der späteren Hieroglyphenschrift zugrundeliegen (neben der - elementaren - semographischen (logographischen) Komponente die phonographische; neben der - elementaren - Notation auch die Kennzeichnung). Eine genauere schriftgeschichtliche Analyse der frühen Schriftzeugnisse findet sich bei Kahl 1994.

Wolfgang Helck hat neuerdings die Frage aufgeworfen, ob es neben und vor der hier in Rede stehenden Hieroglyphenschrift in Ägypten noch bzw. schon eine andere Schrift gegeben habe, deren Heimatgebiet Unterägypten sei, eine Schrift, die er nach dem prädynastischen Zentralort Buto als „butische Schrift" bezeichnet (Helck 1985; 1987). Er führt hier vor allem die zahlreichen Schriftzeichen der ältesten — oberägyptischen Schrift ins Feld, die auf der Basis der späteren Hieroglyphenschrift nicht gelesen werden können. Da jedoch auch eine „,butische“ Lesung nach einem „butischen“" Schriftsystem (evtl. als Verschriftlichung einer anderen als der ägyptischen Sprache) kaum über die eine oder andere Vermutung hinaus kommt, muß die Frage unentschieden bleiben. Ein anderes Indiz liegt möglicherweise in den durch die Annalen überlieferten Namen prädynastischer unterägyptischer Könige vor, die zwar mit Zeichen geschrieben sind, die der Form nach solche der späteren Hieroglyphenschrift sein könnten, die aber samt und sonders nicht ägyptisch gelesen werden können (was allerdings allein daran liegen könnte, daß die Na- men keine ägyptischen wären). Schließlich werden Topfmarken, vor allem aus der frühen dynastischen Zeit, angeführt (Helck 1990), die auf Beigabegefäßen sowohl in Oberägypten als auch in Unterägypten bezeugt sind, die nach der Art ihrer Kombinierbarkeit den Eindruck von Schrift erwecken, deren Zeichenformen aber so wenig mit der späteren Hieroglyphenschrift $\mathrm{zu}$ tun haben, daß diese, wenn es sich in der Tat um Schrift handelt, eine andere als die spätere Hieroglyphenschrift sein muß. Da diese Art von Topfmarken jedoch erst zu einer Zeit geläufig ist, als es die Hieroglyphenschrift bereits gab, könnte es sich um Schrift-Imitate handeln, mit denen unterägyptische Töpfer ihren Produkten einen höheren Prestige- und damit Verkaufswert zu geben suchten. Für diese Interpretation spricht nicht zuletzt die Diskrepanz zwischen der hohen keramischen Qualität und der unsicheren Formgebung bei den Topfmarken.

Die vielberufenen Querverbindungen nach Sumer (oder Elam) lassen sich unter derzeitigen Gegebenheiten schwer beurteilen. Es ist durchaus wahrscheinlich, daß die Schriftentwicklung hier und dort nicht unabhängig voneinander geschah. Die genaueren Zusammenhänge lassen sich jedoch noch kaum definitiv bestimmen. Sicher ist, daß sich die sumerische Keilschrift und die ägyptische Hieroglyphenschrift asynchron entwickelt haben. Wenn etwa die sumerische Keilschrift tatsächlich, wie die jeweils ältesten überlieferten Anfänge bzw. Vorgänger anzunehmen gestatten, der ägyptischen Hieroglyphenschrift voranging, so wurde sie in der frühen dynastischen Zeit in der Entwicklung der zukunftsträchtigen phonographischen Komponente überholt, die in Ägypten früher systematisch ausgebaut wurde als in Sumer (Schenkel 1983, 54-57).

\section{Einzelentwicklungen: \\ Zeichenvorrat, Verhältnis Notation zu Kennzeichnung, Silbenschrift}

5.1. Der Zeichenvorrat der Hieroglyphenschrift ist in ständiger Veränderung begriffen. Dramatisch zu nennen sind diese Veränderungen jedoch nur in der Frühzeit und in den späten, ptolemäisch-römischen Tempelinschriften. In der frühen Zeit kommen viele Schriftzeichen außer Gebrauch, andere werden neu eingeführt. Der Umschichtungsprozeß führt zu einem Zeichenvorrat, der seit dem Mittleren Reich (Anfang 2. Jahrtausend 
v. Chr.) einen Maximal-Umfang von mehr als 1000, aber weniger als 2000 Zeichen hat, von denen deutlich weniger als 1000 im allgemeinen Gebrauch stehen (die Standardzeichenliste bei Gardiner 1927 enthält weniger als 800). In den späten Tempelinschriften wächst die Zahl auf viele Tausende an. - Bei der Erweiterung des Inventars spielt die Bildhaftigkeit der Schrift eine entscheidende Rolle: Die Möglichkeit, neue Semogramme zu schaffen, steht von daher stets offen. Aus Semogrammen können generell - nach dem Rebusprinzip - Phonogramme abgeleitet werden. Extensiv nutzen diese Möglichkeit die Tempelinschriften der griechisch-römischen Zeit. Daneben wird gelegentlich, besonders im spätesten der großen Tempel, dem von Isnā, das Prinzip der Akrophonie (maßgebend ist der wortanlautende Konsonant) zur Gewinnung von Phonogrammen herangezogen (Sauneron 1982), ein Prinzip, das in älterer Zeit bereits für kryptographische Zwecke genutzt wurde.

5.2. Im Verhältnis Notation - Kennzeichnung sind Verschiebungen $\mathrm{zu}$ beobachten. Während man in der frühen Zeit des Schriftgebrauchs oft die Notation als das Zentrum betrachtet, um das herum Kennzeichnungen angeordnet werden können (Reihenfolge: Kennzeichnung(en) - Notation - Kennzeichnung(en)), bevorzugt man später eine unidirektionale Abfolge der beiden Arten von Zeichen (Reihenfolge: Notation - Kennzeichnung(en)). Seit der Schriftreform des Mittleren Reiches (ca. 2000 v. Chr.) kann als Standard-Verfahren der Morphem-, Wortund Wortformschreibung die Reihenfolge Phonographische Notation(en) - allgemeinsemographische Kennzeichnung(en) gelten. Es werden jedoch - aus diversen Gründen - viele ältere orthographische Einzellösungen nach anderen Prinzipien weiter benutzt. Bei den Semogrammen tritt jetzt eine Konzentration auf allgemeine Determinative ein, es wird eine Art semantisches Klassifikationssystem (siehe 2.2.5.) entwickelt. - Verwässert wird nach und nach das Prinzip Notation und Kennzeichnung in den Kursiven, so besonders ausgeprägt im Demotischen, wo die Funktion der Einzelzeichen hinter den gesamthaften Morphem- oder Wortbildern zurücktritt.

\subsection{Silbenschrift}

Im Neuen Reich (18.-20. Dynastie, ca. 15.12. Jahrhundert v. Chr.) steht, vorzugsweise für die Schreibung vorderasiatischer (semitischer, genauer: alt-kanaanäischer) Fremdwörter, aber auch fallweise für die Schreibung ägyptischer Wörter eine rudimentäre Silbenschrift in Gebrauch, die aus der StandardHieroglyphenschrift hervorgegangen ist und mit dieser zusammen benutzt wird (zuletzt Schenkel 1986, Helck 1989, Schneider 1992, Zeidler 1993). Der Unterschied zur StandardHieroglyphenschrift besteht darin, daß die Silbenschrift - bis zu einem gewissen Grade - explizit die Vokale schreibt, die in der Standard-Hieroglyphenschrift ungeschrieben bleiben. Rudimentär ist diese Silbenschrift insofern, als nicht alle Vokale der ägyptischen (oder auch der alt-kanaanäischen) Sprache eindeutig geschrieben werden. Unterschieden werden i/e (fallweise auch Sonderschreibung für $e$ ) $u / o$ (fallweise auch Sonderschreibung für o) und die restlichen Vokale, worunter $a$, Schwa mobile (a) und Schwa quiescens ( $\emptyset$ ) fallen. - Die Silbenschreibung folgt — über das Standard-Hieroglyphenschrift-Prinzip hinaus, das nach Gelb (1963) ebenfalls als Silbenschrift zu interpretieren ist - zwei Prinzipien, dem „Keilschrift-Prinzip“ und dem „Devanagari-Prinzip“. Silbenzeichen nach dem Keilschrift-Prinzip stellen eine Silbe en bloc dar. Vielfach handelt es sich um Schreibungen für ägyptische Wörter mit entsprechender Lautform, die nach dem Rebus-Prinzip für beliebige gleich oder ähnlich lautende Silben eingesetzt werden. Beispiele: I hu (eine Schreibung für das Wort *hǒ < *hă ; ,tausend"), 年 $b i$ (eine Schreibung für $* b i<*$ bi; „Ba (eine Personenkonstituente)“). Silbenzeichen nach dem Devanagari-Prinzip bestehen aus einer oder zwei Komponenten, entweder einer Komponente, die den silbenanlautenden Konsonanten bezeichnet, gefolgt von einer zweiten Komponente, die einen der Vokale i/ $e$ (evtl. $e$ ), u/o (evtl. o) bezeichnet, oder aus der den silbenanlautenden Konsonanten bezeichnenden Komponente allein, in welchem Falle der folgende Vokal als $a$, $\partial$ oder $\emptyset$ anzunehmen ist. Beispiele: $\square h+\emptyset$ für $h a, \nabla w$ $h+i$ (ursprünglich $i$ ) für $h i$, ø』 $h+u$ (ursprünglich $w$ ) für $h u$. - Die Silbenzeichen nach dem Devanagari-Prinzip bezeichnen stets offene Silben, stellen mithin — nach der Silbenstruktur des Ägyptischen - Buchstabiersilben dar. Die Anfänge dieser Schreibweise gehen mindestens bis ins Mittlere Reich (Anfang 2. Jahrtausend v. Chr.) zurück. Sie basiert - ähnlich wie die Plene-Schreibungen semitischer Schriften - auf Neuinterpretation von Schreibungen im Anschluß an lautliche Veränderungen des damit Geschriebenen. So ist fallweise deutlich, daß Schreibun- 
gen für Silben mit $u$ auf Schreibungen für geschlossene Silben mit schließendem Konsonanten $w$ zurückgehen. Versteht man die Schreibung der Konsonanten nach dem Standard-Hieroglyphenschrift-Prinzip als Silbenschreibung, so handelt es sich im übrigen bei der Silbenschrift nach dem Devanagari-Prinzip um eine Variante des Standard-Prinzips Notation + Kennzeichnung - Notation einer Silbe unter fallweise expliziter Kennzeichnung bezüglich der vokalischen Komponente. - Bei der Ausgestaltung der Silbenschrift im Neuen Reich dürfte wohl die zeitgenössische Keilschrift Pate gestanden haben, in der die internationale Korrespondenz geführt wurde, selbst in Ägypten. - Im Laufe der 20. Dynastie (12. Jahrhundert v. Chr.) beginnt die Silbenschrift zu verwildern, möglicherweise als Folge gravierender Lautentwicklungen bei den Vokalen, die die Verbindung zu den geltenden Schreibungen zerstörten.

Eine ähnliche Silbenschreibung läßt sich in der späten Zeit, namentlich in der Ptolemäerund Römerzeit, beobachten, vor allem in den Transkriptionen griechischer Namen. Hier werden sehr häufig explizit die Vokale $u / o$ und i/e geschrieben, oft, aber deutlich weniger häufig, der Vokal $a$. Für das ptolemäische Demotisch liegt eine eingehende Untersuchung von Willy Clarysse vor (Clarysse \& Van der Veken 1983). Ihre Resultate lassen sich — nach Einschätzung Heinz-Josef Thissens - auf das Demotische insgesamt verallgemeinern und darüber hinaus - nach eigener Einschätzung — auch auf das Hieroglyphisch-Ägyptische übertragen. (Die Beobachtung findet sich bereits bei Schwartze (1843), der die Hälfte seines verschrobenen 2000-Seiten-Werkes der Frage widmet, ob die Hieroglyphenschrift eine Silbenschrift sei und dabei richtig bemerkt, daß unter den Vokalen, die im Prinzip alle geschrieben werden können, $i$, $o$ und $u$ bevorzugt geschrieben werden, siehe etwa 489.) Auch wenn die Vokalschreibung Ähnlichkeit mit der Silbenschreibung des Neuen Reiches hat, fragt es sich dennoch, ob hier überhaupt ein Zusammenhang besteht, ob nicht vielmehr - ein Vorschlag Klaus Beyers - die zeitgenössische aramäische Schrift Pate stand, deren Vokalschreibung, über die hier nicht im einzelnen referiert werden kann, mit der späteren ägyptischen durchaus vergleichbar ist $(\rightarrow$ Art. 20). Die Frage muß im übrigen im weiteren Rahmen gesehen werden, im Rahmen nämlich der Schriften, die in den letzten vorchristlichen
Jahrhunderten das Devanagari-Prinzip anwenden. Einzubeziehen wären hier namentlich die persische Keilschrift und die meroitische Schrift mit ihren besonderen Beziehungen zur ägyptischen Hieroglyphenschrift (zu letzterer Weiteres unter 6.).

\subsection{Versuche in phonographischer Alphabetschrift}

In der Saitenzeit (6./7. Jahrhundert v. Chr.), $\mathrm{zu}$ einer Zeit, in der sich die Kontakte zwischen Ägyptern und Griechen auf ägyptischem Boden intensivierten, finden sich Texte, die zu Notationen mit Einkonsonantenzeichen und ohne Determinative tendieren. Auch wenn man ähnliche Schreibweisen in Texten aus dem Alten Reich anführen kann und obwohl gerade die Saitenzeit ein Faible für alte Texte hat, sprechen die auf diesem Wege erzielten Orthographien bei genauerer Betrachtung eher für Beeinflussung durch die griechische Alphabetschrift als für innerägyptischen Archaismus (siehe Gunn 1943, 55-56).

\section{Abhängigkeit anderer Schriften von der ägyptischen}

Bei der Entwicklung der westsemitischen Alphabetschrift(en) (proto-sinaitisch, proto-kanaanäisch) hat offensichtlich, was das Prinzip angeht, die ägyptische Hieroglyphenschrift (Standard-Prinzip) Pate gestanden. Hier wie dort gibt die Schrift über Konsonanten Auskunft, nicht aber über Vokale, wogegen die andere Schrift, die als Vorbild in Betracht gekommen wäre, die Keilschrift, von den Vokalen nicht absieht. Dagegen ist die Herleitung der Zeichenformen aus ägyptischen Kursiv-Zeichen, die bereits im 19. Jahrhundert beliebt war und durch die Entdeckung der proto-sinaitischen Inschriften - das geographische missing link - im 20. Jahrhundert noch einmal Oberwasser erhielt, problematisch ( $\rightarrow$ Art. 20, 29). Die früheren Versuche leiden darunter, daß sie ihre Vergleichsobjekte aus viel zu späten Zeiten wählen (Sass 1988, 135-168; 1991, 4-27). Die Zeichenformen könnten auch originale Erfindungen nach dem Rebusprinzip darstellen (hierzu auch Beyer 1984, 72-76).

Umgekehrt liegen die Dinge bei der meroitischen Schrift, die spätestens im ausgehenden 3. Jahrhundert v. Chr. entstanden ist. Dieser, die in einer Kursivschrift und in einer „Hieroglyphenschrift“ vorliegt, liegen ägyptische Zeichenformen zugrunde, die sich im 
Falle der Kursive in der Hauptsache auf den frühdemotischen Schriftduktus (ca. 2. Hälfte des 6. Jahrhunderts v. Chr.) zurückführen lassen (Priese 1973). Dagegen ist sie ihrem Prinzip nach eine Silbenschrift des DevanagariTyps, die anders als die ägyptische Silbenschrift des Neuen Reiches nur $a$ durch Nichtschreibung wiedergibt, dagegen Schwa mobile (in meroitistischer Transkription $e$ ) und Schwa quiescens $(\emptyset)$, wenn auch - wie etwa die äthiopische Schrift - mit dem gleichen Zeichen, explizit schreibt (Hintze 1973, 1987). Ein genetischer Zusammenhang mit der ägyptischen Silbenschrift ist derzeit zwar nicht definitiv auszuschließen, jedoch nicht sehr wahrscheinlich. Plausibler ist ein Zusammenhang mit anderen zeitgenössischen Schriften, zumal der aramäischen oder der keilschriftlich-persischen, zu denen dann auch die späte Silbenschrift in Ägypten in Beziehung zu setzen wäre (vgl. unter 5.4.).

Ägyptische Zeichenformen wurden im Rahmen der Wiedergabe des Ägyptischen in griechischer Alphabetschrift (für die Wiedergabe vor allem solcher Laute, für die das Griechische kein geeignetes Äquivalent besitzt) benutzt (siehe etwa Osing 1976, 6-7), auf Dauer in Gestalt der sog. Zusatzbuchstaben des koptischen - im Kern griechischen - Alphabets (siehe etwa Gardiner 1927, $5-6)$.

\section{Aussterben}

Die Fähigkeit, Texte in Hieroglyphenschrift zu schreiben, läßt im 2. Jahrhundert n. Chr. deutlich nach, zu einer Zeit, als die demotische Kursive noch beherrscht wird. Die letzte datierbare hieroglyphische Inschrift stammt von 394 n. Chr., die letzte datierbare demotische von $452 \mathrm{n}$. Chr. Beide befinden sich in Philae, dem südlichen Rückzugsgebiet des ägyptischen Kultes, wo dieser noch bis 535 n. Chr. vom Verbot paganer Kulte praktisch ausgenommen war. Im nördlichen Alexandria könnte es zum mindesten bis $391 \mathrm{n}$. Chr. noch Personen gegeben haben, die Hieroglyphen lesen konnten.

\section{Literatur}

Anonymus (ed.). [1973]. Textes et langages de l'Egypte pharaonique. Kairo, I, 1-77.

Beyer, Klaus. 1984. Die aramäischen Texte vom Toten Meer. Göttingen.

Clarysse, W. \& Van der Veken, G. 1983. The Eponymous Priests of Ptolemaic Egypt. Chronological
Lists of the Priests of Alexandria and Ptolemais with a Study of the Demotic Transcriptions of their Names. Leiden [hierzu ergänzend Heinz-Josef Thissen in Enchoria 13, 1985, 179].

Fischer, Henry G. 1977 a., The Orientation of Hieroglyphs. Part I. Reversals. New York.

—. 1977 b. Hieroglyphen. In: Helck, Wolfgang \& Westendorf, Wolfhart (ed.), Lexikon der Ägyptologie. Wiesbaden, II, 1189-1199.

—. 1986. L'écriture et l'art de l'Egypte ancienne. Quatre leçons sur la paléographie et l'épigraphie pharaoniques. Paris.

Gardiner, Alan H. 1927. Egyptian Grammar. Oxford [3. Aufl. London 1952].

Gelb, Ignaz J. 1963. A Study of Writing. 2 Chicago [1. Aufl. 1952].

Gunn, Battiscombe. 1943. Notes on the Naukratis Stela. Journal of Egyptian Archaeology 29, 55-59.

Helck, Wolfgang. 1985. Gedanken zum Ursprung der ägyptischen Schrift. In: Posener-Kriéger, Paule (ed.), Mélanges Gamal Eddin Mokhtar. Kairo, I, 395- 408 .

-. 1987. Untersuchungen zur Thinitenzeit. Wiesbaden.

—. 1989. Grundsätzliches zur sog. „Syllabischen Schreibung“. Studien zur altägyptischen Kultur 16, $121-143$.

—. 1990. Thinitische Topfmarken. Wiesbaden.

Hintze, Fritz. 1973. Some Problems of Meroitic Philology. Meroitica 1, 321-336.

- 1987. Zur Interpretation des meroitischen Schriftsystems. Beiträge zur Sudanforschung 2, $41-50$.

Kahl, Jochem. 1994. Das System der ägyptischen Hieroglyphenschrift in der 0.-3. Dynastie. Wiesbaden.

Kaiser, Werner. 1990. Zur Entstehung des gesamtägyptischen Staates. Mitteilungen des Deutschen Archäologischen Instituts Abteilung Kairo 46, 287-299.

Kees, Hermann (ed.). 1959. Ägyptische Schrift und Sprache. In: Handbuch der Orientalistik, Leiden.

Osing, Jürgen. 1976. Der spätägyptische Papyrus BM 10808. Wiesbaden.

Priese, Karl-Heinz. 1973. Zur Entstehung der meroitischen Schrift. Meroitica 1, 273-306.

Sauneron, Serge. 1982. Esna VIII. Kairo.

Sass, Benjamin. 1988. The Genesis of the Alphabet and its Development in the Second Millenium B. C. Wiesbaden.

—. 1991. Studia alphabetica. Freiburg (Schweiz)/ Göttingen.

Schenkel, Wolfgang. 1981. Rebus-, Buchstabiersilben- und Konsonantenschrift. Göttinger Miszellen $52,83-95$. 
—. 1983. Wozu die Ägypter eine Schrift brauchten. In: Assmann, Aleida, Assmann, Jan \& Hardmeier, Christof (ed.), Schrift und Gedächtnis. München, 45-63.

—. 1984. Schrift. In: Helck, Wolfgang \& Westendorf, Wolfhart (ed.), Lexikon der Ägyptologie. Wiesbaden, V, 713-735.

- 1986. Syllabische Schreibung. In: Helck, Wolfgang \& Westendorf, Wolfhart (ed.), Lexikon der Ägyptologie. Wiesbaden, VI, 114-122.

Schneider, Thomas. 1992. Asiatische Personennamen in ägyptischen Quellen des Neuen Reiches. Freiburg (Schweiz)/Göttingen.
Schwartze, Moritz G. 1843. Das alte Aegypten ... Erster Theil. Darstellung und Beurtheilung der vornehmsten Entzifferungs-Systeme der drei altägyptischen Schrift-Arten. Erster Theil. Leipzig [XLVIII, 2183 S.; mehr nicht erschienen].

Winter, Erich. 1989. Hieroglyphen. In: Reallexikon für Antike und Christentum. Stuttgart, Lief. 113, 83-103.

Zeidler, Jürgen. 1993. A New Approach to the Late Egyptian „Syllabic Orthography“. In: Sesto Congresso Internazionale di Egittologia. Turin. III, $579-590$.

Wolfgang Schenkel, Tübingen (Deutschland) 